\title{
DÉFAUT DE JUSTICE ET NARRATIONS COMPENSATOIRES CHEZ SOPHIE BIENVENU, LYDIE SALVAYRE ET JULIA DECK \\ Cassie Bérard $^{1}$ et Elise Warren ${ }^{2}$
}

RESUMÉ : Cet article veut observer le rôle déficient de la justice dans trois œuvres de fiction narrative contemporaines où le personnage principal est accusé d'un crime. Silencieuse ou stéréotypée, la justice représentée dans les romans n'en

\footnotetext{
${ }_{2}^{1}$ Professeur (Université du Québec à Montréal) - mail : m_degos@yahoo.fr

2 Étudiante à la maîtrise (Université du Québec à Montréal) - mail : m_degos@yahoo.fr
} 
impose pas moins une vérité de la fiction (un verdict de culpabilité) qui influence la lecture. Ainsi, nous cherchons à voir comment les narrations ambiguës tentent de compenser le défaut de justice et comment, par une lecture réparatrice, le lecteur redonne aux personnages une humanité que la justice leur retire.

MOTS-CLÉS: justice, narrations, vérité, empathie, surinterprétation.

\section{LACK OF JUSTICE AND COMPENSATORY NARRATIONS IN THE NOVELS OF SOPHIE BIENVENU, LYDIE SALVAYRE AND JULIA DECK}

ABSTRACT: This article seeks to observe the deficient role of justice in three contemporary narrative fictional works where the main character is accused of a crime. Silent or stereotyped, justice represented in novels nonetheless imposes a truth of fiction (a verdict of guilt) that influences reading. Thus, we try to see how ambiguous narratives compensate for the lack of justice and how, through a restorative reading, the reader gives back to the characters a humanity that justice withdraws from them.

KEYWORDS : justice, narrations, truth, empathy, overinterpretation.

Les processus de justice appellent à la vérité, à ce « désir du fait vrai », or ils se trouvent couramment confrontés à son impossibilité : à un flou, une nébulosité, une indétermination. Lorsqu'on amorce une analyse des conduites narratives en situation judiciaire dans les œuvres de fiction, force est de constater que de suspects en témoins s'exprimant, il n'est pas chose aisée d'établir une vérité objective à travers ce qui se présente plus souvent comme un tissu discursif de dissimulations, d'omissions, de mensonges et d'oublis. De fait, le principe de vérité s'avère fuyant dans les fictions judiciaires, et se donne comme illusion, à condition de considérer, comme Michel Butor, que toute prise de parole, toute narration «se propose à nous comme un rythme de pleins et de vides » (BUTOR, $2006: 441$ ). Toute narration n'atteint à une forme de vérité que de manière partielle, et plus encore impressionniste lorsqu'il s'agit de récits où la crédibilité du narrateur est mise en doute - car il n'est pas rare qu'elle le soit lorsque la justice s'implique (ou s'applique). De manière partielle, disions-nous, puisque la narration ne peut que présupposer, ainsi que l'annonce Lubomír Doležel, « the independent existence of a world to which the corresponding sentences refer, fail to refer, or pretend to refer³. »(DOLEŽEL, 1998 : 146). Si une vérité de la fiction est ainsi envisageable, elle ne peut qu'être fabulée par le lecteur à partir de ce que le récit offre d'informations

\footnotetext{
${ }^{3}$ " l'existence indépendante d'un univers (fictionnel) auquel les énoncés renvoient, auquel ils n'arrivent pas à renvoyer ou auquel ils prétendent renvoyer. » (Notre traduction.)
} 
minimales ou détournées. Et donc, accéder à cet univers de référence objectif ou croire y accéder en tant que lecteur, alors même que la narration des récits est suspecte, pointe moins la vérité que les mondes possibles auxquels nous convie chaque prise de parole, lesquels sont autant de voies d'accès vers l'expérience du soupçon. Si le lecteur, consentant à suspendre son incrédulité comme l'exprime Samuel Coleridge, accepte de se laisser duper, les instances judiciaires représentées au sein des fictions sont toutefois, quant à elles, rarement dupes. Leur conscience, voire leur préoccupation, quant aux enjeux de responsabilité, d'implication et d'intérêt les place en situation d'autorité face aux témoins ou aux suspects appelés à la barre, en ce qu'elles exercent une fonction officielle. Nous observons que ces instances judiciaires représentées sont dotées d'un pouvoir décisionnel, et se configurent comme des forces manipulatrices capables de faire advenir la vérité là où il n'y a que confusion, quitte à contrôler cette vérité. Pour pallier ce contrôle autoritaire qui agit sur leur destinée autant que sur notre jugement de lecteur, les personnages de fiction soumis à la justice se trouvent sans moyen autre que la parole. Leur discours fait ainsi contrepoids à la vérité établie par la justice. Aussi, de la tension entre justice et littérature ressortent, croyons-nous, des narrations compensatoires, des narrations qui résistent à l'ordre des choses, aux apparences, et qui font le choix de la confusion, le choix du désordre, de la désobéissance. Bien entendu, cette désobéissance relève aussi d'une position du lecteur, forcé qu'il est, par ses propres présupposés, de s'en remettre la plupart du temps à l'autorité judiciaire dans l'interprétation qu'il propose des œuvres, dans sa compréhension des événements. Aussi ose-t-il un geste interprétatif audacieux lorsqu'il s'oppose à l'autorité en place dans la fiction afin de redonner aux narrateurs leurs pleins droits, leur autonomie ou leur innocence. C'est en pratiquant une lecture réparatrice, voire revendicatrice d'une empathie qui fait défaut aux instances judiciaires, que nous en viendrons - tel est l'objectif de cet article - à sauvegarder la pleine humanité des narrateurs et narratrices qui déambulent, dans les romans Et au pire, on se mariera de Sophie Bienvenu, La puissance des mouches de Lydie Salvayre et Viviane Élisabeth Fauville de Julia Deck, à travers les filets d'une justice, au moins en apparence, insensible.

\section{DÉFENSE PAR FABULATION, DÉFENSE PAR DIGRESSION}

Le roman Et au pire, on se marierase présente sous la forme d'un dialogue dont il nous est donné à lire une seule des deux voix, celle d'une jeune fille de treize ans qui se nomme Aïcha. Elle nous apprend, tandis qu'elle se livre maladroitement, sans ménager mots crus et obscénités, que sa mère est une " folle » qui n'agit que pour lui mettre des bâtons dans les roues. Aussi, selon Aïcha, si sa mère fait fuir son amant, ce n'est pas parce qu'il abusait de sa fille en la soumettant à des pratiques sexuelles dont l'enfant ne pouvait mesurer la portée, mais bien parce 
que sa mère est jalouse. N'avoir aucune idée de la portée des gestes et des paroles, voilà ce qui caractérise le personnage d'Aïcha, et le lecteur a tôt fait de s'en rendre compte. C'est une immaturité qui se dégage de cette narration naïve; Aïcha ne rationalise pas, elle se décharge. Décharge de colère, de mépris, sa parole est un flot saccadé d'insultes et d'énoncés improbables qui lui permettent de relater les événements à sa façon, de les façonner avec sa fureur d'enfant.

Plus d'une fois, en effet, Aïcha revient sur sa version des faits. Plus d'une fois elle admet n'avoir pas agi tel qu'elle vient de le décrire. Sa narration est pavée d'allers-retours : «Non, OK, c'est pas vrai, j’ai pas crié ça. Mais ça aurait été le fun. J'aurais voulu crier ça. Si on avait frenché pour vrai, je pense que je l'aurais fait. Sauf que là, non. » (BIENVENU, 2015 : 23.) Elle semble concevoir la situation dans laquelle elle est impliquée comme un jeu, elle ne prend pas au sérieux son allocutaire, elle énonce sa vie en fictions, en fantasmes, en inventions.

La situation ne se présente pourtant pas comme un jeu. Un affrontement plutôt, entre deux personnages aux moyens inégaux. Et si les enjeux ainsi que l'issue de ce duel restent incertains, la cause, elle, s'éclaire à mesure que la jeune fille se dévoile. Il est question d'une relation amoureuse illégale. Un homme, Baz, du double de son âge. Des débordements. Aïcha est placée sous interrogatoire. Le récit ne nous dit pas d'emblée ce que cela implique, mais laisse supposer que des complications sont survenues, que l'aventure amoureuse ne s'est pas bien terminée. Or là se joue toute la tension du récit. Ces complications, elles sont relatées par la narration d'Aïcha, mais l'on sait qu'Aïcha ne se rend pas compte de la portée de ces complications. Il est donc impossible de s'accrocher à l'un ou à l'autre des énoncés de la narration, car chacun est potentiellement une fabulation. Aussi est-il opportun de parler de "moyens inégaux » lorsque vient le temps de s'intéresser aux rôles que jouent, dans cet interrogatoire, l'une et l'autre des parties. Si, d'un côté, l'allocutaire (l'interrogatrice) est privée de la voix; de l'autre, la narratriceest privée de bon sens - et de toute autorité sur sa propre parole. Mais de cette lacune, dirons-nous, la justice n'en a que faire.

Dans La puissance des mouches,le même procédé d'effacement de l'allocutaire est observé. La voix de la justice est escamotée, et pourtant, on ne peut échapper à sa présence influente, puisqu'elle reçoit le discours du narrateur en abondance, fragments expansifs, digressions. De fait, la justice ne se prononce pas avec des mots, mais plutôt, elle est l'oreille qui entend, elle est l'instance qui autorise ou condamne. Le personnage principal, un homme de quarante-huit ans qui ne cache ni sa mesquinerie ni sa vanité, s'adresse tour à tour, le temps de logorrhées déroutantes, à un juge, à monsieur Jean (un infirmier de prison), au docteur Vile motte, puis à l'avocat de sa défense. Il aborde des banalités, parle de ses relations tendues avec sa femme qu'il trouve insupportable, de l'admiration qu'il cherche à obtenir de son patron, il parle de son rôle de guide au musée, et il parle de Blaise Pascal, à partir duquel il philosophe et argumente; Blaise Pascal est le porte-étendard de 
ses aptitudes à penser. De fait, le narrateur pense, il ne cesse jamais de penser, de parler, de se diagnostiquer, si bien que ces pensées pêle-mêle deviennent la toile tendue aux allocutaires, sa propre défense.

Sous procès, il est appelé à témoigner à titre d'accusé. Il consacre l'essentiel de son discours à étaler ses défauts et à faire l'apologie de Blaise Pascal. Il faut ainsi attendre la dernière ligne du roman pour comprendre qu'il est coupable du meurtre de son père. Acte abominable qu'il a la décence de ne pas nier. De fait, le narrateur se déclare monstrueux et cruel et il cerne avec lucidité le moment, dans son cheminement personnel, où il est devenu criminel. Toutefois, il ne se conçoit pas comme un méchant authentique et, dans son procès, insiste pour que soit relativisée sa part de méchanceté. Si son père est un "méchant par vocation », c'est ce qu'explique le narrateur en élaborant une typologie, il n'est, quant à lui, qu'un «méchant occasionnel » (SALVAYRE, 1995 : 108). Tout son témoignage tend ainsi, comprenons-nous, à prouver cette thèse: ne pas être un criminel véritable, mais « être devenu » criminel, par la force des choses. Le lecteur, auditeur privilégié, n'est pas sans réagir à ces confessions; elles organisent sa lecture. Quelle est leur nature, leur visée? Derrière un homme en apparence vaniteux se profile un autre homme, sensible, accablé par un héritagedeviolence. Cette image en filigrane interpelle, de même, la sensibilité du lecteur. Mais qu'est tenue de faire la justice devant un tel aveu?

Aïcha s'adresse à une femme dont le roman Et au pire, on se mariera occulte l'identité. L'image que le lecteur se forme de cette allocutaire reste donc floue, incertaine; tracée, nécessairement, par les mots de la narratrice : "C'est drôle comment t'écarquilles les yeux, des fois. " (BIENVENU, 2015:31); "Pourquoi t'es méchante? »(BIENVENU, 2015 : 89.) Des réactions de la jeune fille, le lecteur déduit toutefois qu'il s'agit d'une intervenante sociale, d'une psychologue ou encore d'une agente de police qui l'aura prise en charge alors qu'elle vient d'être arrêtée. Cette allocutaire, qui incarne la justice dans le roman, cherche à soutirer à Aïchades informations, à faire émerger sa version des faits. D'emblée, par son retrait, ou plutôt par son absence dans le texte, la justice représentée ne prend pas réellement part au récit; elle l'oriente mais ne le constitue pas. Elle reste dans l'ombre, sans agir. Néanmoins, par la puissance de son silence, elle influence manifestement les perceptions du lecteur, et elle exauce une vérité de la fiction que le récit d'Aïcha n'admet pas forcément puisqu'au contraire il s'efforce de la contester : la culpabilité de Baz dans toute cette affaire. Affaire qui dépasse, réalisons-nous tardivement, les rapports sexuels entre un homme majeur et une jeune mineure pour se transformer en sordide crime passionnel. Une femme, Élisanne Blais, est retrouvée morte dans le lit de Baz. L'a-t-il tuée ou est-ce Aïcha la coupable?

$\mathrm{Au}$ fond, c'est une enfant en train de se construire une image de soi que nous observons dans Et au pire, on se mariera, et non une parole en train de se commettre. Car il faut être clair : malgré ses tergiversations multiples, Aïcha reste fer- 
me sur un point : il n'y a qu'elle qui soit coupable. Pour le lecteur, cette déclaration, qui revient de manière insistante, révèle une faille chez la jeune fille, dont elle ne prend pas pleine conscience. Pour le lecteur, déceler cette faille, la laisser sillonner la lecture, revient à redonner à la narratrice cette humanité que la justice lui enlève en lui soutirant de force une confession. Au contraire des narrateurs indignes de confiance qui attirent la méfiance, soutient Greta Olson, les narrateurs faillibles attirent l'empathie. Le lecteur est porté à s'y identifier, dans la mesure où il compose avec des erreurs naturelles auxquelles nul personnage ne peut non plus échapper :

fallible narrators do not reliably report on narrative events because they are mistaken about their judgments or perceptions or are biased. Fallible narrators' perceptions can be impaired because they are children with limited education or experience, [or] their reports can seem insufficient because their sources of information are biased and incomplete ${ }^{4}($ OLSON, 2003: 101).

Olson associe la faillibilité des narrateurs à des causes circonstancielles qui influencent leur comportement, leurs réactions et, de là, leur narration. Elle pointe les « capacités restreintes » des narrateurs qui se trouvent à agir dans des situations particulières qu'ils sont vraisemblablement inaptes à gérer. Le personnage de l'enfant, pour Olson, est un exemple frappant. Généralement naïf car peu instruit, peu expérimenté, il n'est pas en mesure, dans certaines circonstances, de comprendre la subtilité des rapports sociaux, des rites et politesses d'usage ou de saisir la portée de certaines expressions et actions. Son récit est alors marqué par une incapacité à percevoir adéquatement les situations et, du coup, à les décrire de manière à ce qu'elles soient conformes à la réalité. En ce sens, les erreurs commises par ces narrateurs faillibles les prémuniraient de la méfiance du lecteur, sensiblement compréhensif et coopératif dans sa lecture. Si les narrateurs faillibles «make individual mistakes or leave open informational gaps thatneed to befilled in ${ }^{5}$ »(OLSON, 2003:104), le lecteur accepterait de réparer les erreurs et de « remplir les trous » plutôt que de discréditer la parole du personnage.

Cette lecture réparatrice, essentiellement empathique, amène à concevoir le personnage comme un être agissant investi dans son univers, participant aux interactions socio-humaines, bien que fictionnelles. L'approche «based on real-life

\footnotetext{
${ }^{4}$ «les narrateurs faillibles ne sont pas fiables lorsqu'ils rapportent des événements puisque leurs jugements ou leurs perceptions sont fautifs, ou alors ils sont biaisés. Les perceptions des narrateurs faillibles peuvent être altérées parce qu'il s'agit, par exemple, d'enfants dont l'expérience ou l'éducation est limitée, ou alors leurs récits peuvent paraître insuffisants parce que leurs sources d'information sont biaisées ou incomplètes. » (Notre traduction.)

${ }^{5}$ « font des erreurs occasionnelles ou laissent en suspens des informations, laissent des trous qui demandent à être comblés » (Notre traduction.)
} 
parameters ${ }^{6}$ » (NÜNNING, 2005:94) sur laquelle s'appuie une telle lecture fait en sorte que le lecteur tend à analyser le personnage en regard de ses propres balises psychosociales. Il lui importe alors d'élargir sa lecture à une compréhension globale des tensions et motifs qui opèrent dans le comportement du personnage, au-delà de la scène d'énonciation. La situation narrative d'Aïcha place le lecteur dans cette position empathique. Aïcha se révèle, dans toute sa verdeur, sa naïveté et, sans l'ellipse que cause le silence de son interlocutrice, le " procès de lecture » serait simple : une jeune fille tente tant bien que mal de se dire, de se mettre en récit, et il appartient au lecteur de dégager le réel de la fabulation. Toutefois, une interlocutrice se tait; le lecteur n'est pas, au sens où l'entend la pragmatique d'Oswald Ducrot, « l'allocutaire », il est « l'auditeur », « interceptant avec malice, voire avec une curiosité quelque peu malsaine, ce qui est destiné à l'autre, à celle ou à celui dont se recommande l'instance énonciatrice.» (apud SCHUEREWEGEN, 1986 : 217.) Le terme «procès » convient d'autant plus qu'il s'inscrit effectivement, ici, dans un registre judiciaire : l'allocutaire (à qui s'adresse la narratrice) est l'instance appelée à juger de l'énonciation, et l'auditeur, dans le double jeu de la communication et de la justice, semble a priori soumis à ce qu'arrêtera l'allocutaire, d'autant plus que celle-ci détient une autorité légitime. Le lecteur doit se faire violence - doit-il le faire? - pour oblitérer la situation énonciative, pour récuser les paroles accusatrices inférées que l'allocutaire lance, pour absorber les mensonges que cette voix muette détrousse. Difficile, dans un tel contexte, de maintenir l'empathique décodage du discours : cette narration dissimule quelque chose. Cependant, en dissimulant quelque chose, elle se dévoile aussi.

Ce n'est pas le cas de l'allocutaire. En vérité, l'absence de l'allocutaire cause, en ce qui concerne la figure de la justice, une absence de dévoilement : la personne allocutaire ne se révèle pas puisqu'elle n'est pas représentée textuellement. Certes, en revanche, la mainmise de la justice sur le discours d'Aïcha est nette. Parce que cette justice est absente, elle macule l'énonciation de l'enfant en lui donnant tort, l'obligeant à parler pour deux. Il faut qu'Aïcha fasse entendre l'allocutaire, elle ne peut pas se contenter de lui faire entendre sa raison, elle doit de surcroît lui donner sa voix, lui louer sa parole, engendrant un déséquilibre narratif. Par contre, parce qu'elle compense le défaut de justice, la narration porte en elle la tension entre dissimulation et dévoilement qui mettent au jour sa faillibilité. Dans un même élan, la situation énonciative déshumanise l'allocutaire qui s'avère paradoxalement inféodée à une parole à laquelle il reste au moins un pouvoir, celui de transmettre une sensibilité. D'ailleurs cette sensibilité, si elle n'atteint pas son destinataire premier, atteint au moins une cible compensatoire, l'auditeur suppléé, soit le lecteur, et elle l'atteint précisément grâce à l'effacement de l'allocutaire, grâce à l'incapacité de celle-ci à transmettre sa propre humanité, ou encore, son incapacité à transmettre sa capacité à humaniser.

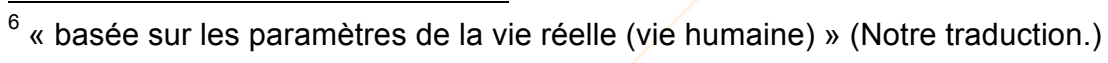


La toile que tisse le narrateur de La puissance des mouches apparait moins fortuite que les fabulations d'Aïcha. Est-ce d'emblée le statut social du personnage qui soulève nos préjugés? Nous pourrions, en effet, nous rallier à la thèse de William Riggan (1981) qui, sans s'embarrasser des nuances, réduit la narration du fou à une dissimulation involontaire; celle du clown, à une dissimulation volontaire visant notamment à provoquer ou à faire rire son public. Dans le même esprit, il y a fort à parier que l'obsession particulière du personnage de La puissance des mouches pour Blaise Pascal, ainsi que son occupation de guide dans un musée, ressurgissent dans sa façon de conduire son discours, dans la surenchère des expressions et les excès de fantaisies. Le narrateur, d'ailleurs, se soucie de ses allocutaires de la même manière qu'il traite les touristes venant au musée : "C'est pourquoi je les guide lentement [...]. C'est pourquoi je les oriente avec tendresse et les entoure de toutes sortes d'attentions. C'est pourquoi je les protège, monsieur Jean, de la peur confuse et enfantine qu'ils éprouvent devant les choses inconnues » (SALVAYRE, 1995 : 55).Ainsi voit-on se construire une parole sensible aux questions humaines, qui ne cesse de s'échapper vers l'enfance, car l'enfance a ceci de tendre et d'attachant, l'enfance est le berceau des peurs confuses et elle convainc des meilleurs sentiments :

J'ai toujours, monsieur Jean, le cœur qui se serre, lorsque je vois arriver un car de tourisme pullman décoré de palmiers aux palmes parfaites. Parce que j'ai l'impression, chaque fois, que ma mère va surgir de l'un d'eux, que je vais la voir descendre, $[\ldots]$ s'accrocher au bras de son mari qui essaie de se dégager de cette charge humaine et qui avance à grandes enjambées dans l'espoir de la semer. (SALVAYRE, $1995: 55)$

La narration dévie manifestement de son mandat véritable pour devenir confidences intimes: «Mais qu'est-ce qui me prend de vous dire tout ça? Je me suis encore éloigné du sujet. Pouvez-vous me rappeler, monsieur le juge, quel est l'objet qui nous occupe? » (SALVAYRE, $1995: 76$.)Or, de manière similaire au soliloque d'Aïcha, ces digressions du personnage, si elles dissimulent l'essentiel des faits qui intéressent la justice, sur un plan intellectuel comme sur un plan émotif, procèdent d'un dévoilement. Le lecteur y reconnait avant tout un homme à la fois capable de rationaliser, donc en contrôle de ses émotions, à la fois qui se sent responsable des gestes qu'il n'a pas eu les moyens de poser enfant, soit à l'époque où il ne pouvait percevoir la réalité avec acuité. Ces gestes qui auraient pu sauver sa mère, sa sœur, mais qui l'auraient mener, lui, au même point, décidément. Car c'est le père, comprenons-nous, qui l'a constitué criminel.

La justice, dans La puissance des mouches, ne se prononce que sous la forme de conséquences : l'homme est reconnu coupable, il se trouve en prison, la nature de la scène d'énonciation nous l'apprend. Les allocutaires sont multiples - juge, infirmier de prison, médecin, avocat; compte tenu de la nature des aveux, ils ne pourront rien pour lui. Or, si le narrateur, justement, tissait sa défense pour un 
autre, pour un auditeur en surplomb ayant une vue d'ensemble? S'il cherchait, audelà des instances judiciaires muettes, impassibles - qui elles, d'ailleurs, ne reçoivent chacune qu'un fragment de son plaidoyer -, un soutien particulier chez le lecteur, capable lui, comme le touriste, de se montrer curieux et de s'émouvoir. De rire même, peut-être : "Je note, à ce propos, que la question du rire ne cesse de m'agiter. » (SALVAYRE, 1995 : 124.)Cela n'expliquerait-il pas ses constantes digressions, ses dérives, les déviations de son discours, les fabrications; le fait qu'il ne s'adresse pas, à proprement parler, à ces allocutaires à qui il feint de s'adresser, mais plus sûrement à l'auditeur contingent qui ne saurait, contrairement à la justice, l'ignorer. De la même manière qu'il lit avec bonheur Pascal, il sait le plaisir que l'on peut retirer d'un voyage de l'esprit : «Faut-il considérer, monsieur Jean, la lecture de Pascal comme un divertissement? » (SALVAYRE, 1995 : 77.) Et peutêtre de la même manière qu'il assume le meurtre de son père, il espère - et ici nous acceptons de surinterpréter - que le lecteur, mieux que la justice, se montrera compréhensif.

Aïcha est-elle coupable, quant à elle, d'avoir tué Élisanne Blais tel qu'elle le raconte : « Je te jure que c'est moi, je te jure! Note-le, que c'est moi, sur ton carnet! C'est la vérité, ce que je t'ai raconté, tout, tout, tout, c'est vrai. » (BIENVENU, 2015 : 147.) Ou est-elle plutôt coupable de mentir pour protéger l'homme qui a tué? Elle est du moins coupable d'amour. D'un amour impossible. Mais la justice parle-t-elle le langage de l'amour? La justice ne parle pas. Aïcha, elle, parle, mais demeure dans l'incompréhension, la confusion. D'où le tragique derrière ce soliloque, du fait que le procédé d'effacement de l'allocutaire, dans Et au pire, on se mariera, porte Aïcha à des actes narratifs excessifs, désespérés, la menant à concevoir une extrême fiction, celle d'un crime qu'elle n'aurait pas commis. Car le verdict est tombé ici aussi : c'est Baz qu'on reconnaît coupable, et l'allocutaire, vraisemblablement, détient (et retient) l'information depuis le début de l'interrogatoire. La justice va jusque-là dans sa cruelle absence : contraindre au mensonge. Pour conjurer le silence, la narration ne peut qu'exagérer, amplifier; l'enfant, s'inventer. Ainsi, la justice silencieuse forme une jeunesse sans repère et impulse un langage sans vérité possible, car la seule vérité que revêt le discours d'Aïcha est celle de l'amour fabulé, de l'amour colmatage, celle du manque, de l'absence, du défaut. Défaut d'attention de la mère, défaut de respect du père. Défaut de parole de l'allocutaire, cette personne trouble que le silence supprime, cette personne qui rassurerait Aïcha et lui dirait que la fiction ne couvrira pas les maux. Mais ce soutien est impossible, nous apprend la justice, représentée par ses impasses narratives. 


\section{nonplus}

\section{L'INNOCENCE, SIGNE DE FOLIE}

Les romans de Sophie Bienvenu et de Lydie Salvayre que nous venons d'analyser présentent un schéma narratif semblable. La justice y occupe une place centrale, dans la mesure où, malgré son silence, elle détermine le contexte d'énonciation; elle détermine l'environnement qui fait émerger la parole. Cette parole s'adresse à un ou plusieurs allocutaires pour atteindre l'auditeur. Dans ce point de contact entre la voix narrative et le récepteur privilégié, avons-nous montré, se noue une relation d'empathie. En acceptant l'immanence de la fiction, cette empathie possible à l'extérieur du texte peut être saisie comme le rempart des narrateurs.

D'une manière différente toutefois, la narration s'emballe dans Viviane Élisabeth Fauville, de Julia Deck, pour placer au centre du récit le personnage féminin à qui le roman doit son titre. D'abord, « vous » êtes Viviane, ensuite " elle » l'est, sans que ce soit à part entière; puis « je », puis «tu », puis « nous » le devenons aussi. Seul le pronom personnel« elles » est épargné dans cette série de déplacements narratifs, mais c'est qu' " elles » regroupent toutes les autres, rendant instable et pluriel le regard posé sur les choses, et multipliant dans le récit les détours, les évitements, les versions. Chez le personnage féminin, ce sont les personnalités qui semblent se multiplier.

Dans le roman de Julia Deck, on nous présente une femme désabusée, prise dans le quotidien monotone auquel la contraint son congé de maternité. Elle vient de tout perdre : son titre prestigieux au travail, son mari, un appartement de luxe, son statut de bourgeoise. Elle n'a que son enfant dont elle parle sans grande émotion. Et puis, tout bascule quand «vous »tuez votre psychanalyste : "Vous plongez la lame juste en dessous de la dernière côte, l'y trempant jusqu'à la garde. Les viscères ont la mollesse du beurre. » (DECK, 2012 : 24.)Les jours suivants, on vous convoque à plusieurs reprises au commissariat de police; vous vous y rendez avec votre enfant, vous restez évasive en répondant aux questions, vous évitez le plus possible de vous incriminer. Lorsque Viviane tente, plus tard dans l'histoire, de tuer Julien, son mari, elle est finalement récupérée par les forces de l'ordre et hospitalisée. À l'hôpital, Viviane confesse son crime : le meurtre du psychanalyste. Or, l'inspecteur relève des incohérences dans son récit. On la traite comme une personne souffrant de maladie mentale. La multiplicité des personnalités narratives résonne ici grandement. D'autant qu'on nous apprend qu'en réalité, un homme du nom de Pascal Planche, un patient du docteur «sous médication depuis sept ans et demi » (DECK, 2012, p. 142), serait l'auteur du meurtre. L'expertise judiciaire confirme. Viviane aurait découvert le corps de son psychanalyste en se présentant à une consultation et, ne sachant comment gérer cet événement, se serait imaginée meurtrière. Effectuant une sorte de transfert, elle se serait créé une nouvelle personnalité, de nouveaux souvenirs, au point d'en affecter la narration du récit. Tour de force esthétique, les procédés narratifs de déplacement, de réticence et de fein- 
te entraînent toutefois la lecture dans des raccourcis interprétatifs qui, estimonsnous, font ressortir certains stéréotypes féminins. Plus précisément en le plaçant, dans le roman, dans la bouche des hommes de loi (agents de police, inspecteur), on fait ressortir le stéréotype de la «femme folle ». La narration éclatée appuie cette image en rendant compte, par procuration, des faits et gestes erratiques du personnage central. La narration opère une contradiction majeure, donnant à lire un récit qui finalement se nie, à la manière de «tout ceci n'était qu'un rêve ». Bientôt, il n'y a plus d'ambiguïté. La fin de l'œuvre est fermée, la trame d'enquête se résout. Les preuves sont là, l'expertise judiciaire s'est prononcée; que "vous » ayez avoué un crime ne change rien, les spécialistes attestent votre innocence. Vous n'êtes pas meurtrière, vous n'êtes que « folle ».

C'est ce qu'on essaie de vous faire croire. Et voilà pourquoi la narration de Viviane Élisabeth Fauville est retorse, selon nous. Et voilà comment elle attaque sournoisement la justice, l'expertise, la vérité représentées. Non pas parce qu'elle désigne faussement une criminelle en détaillant un crime que cette dernière n'a finalement pas commis, paraissant ainsi se jouer du lecteur. Mais bien parce que la narration utilise la folie comme mécanisme pour nous faire croire qu'elle désigne « faussement » une criminelle. Par ce paradoxe complexe qui constitue notre hypothèse, la narration mystifie définitivement le lecteur.

Viviane Élisabeth Fauville est une mère de quarante-deux ans. Elle est coincée dans le rôle que l'on attend d'elle en tant que femme, soumise au déterminisme : «Vous aviez un mari, un travail, un enfant, des obligations qui s'amoncelaient du matin au soir. Les moindres instants de votre existence étaient réglés par la nécessité, et vous voyiez bien qu'il en allait de même pour vos semblables » (DECK, 2012 : 45). À côté de cette réalité, pour pallier la banalité qui les accapare, il ne reste aux femmes qu'à s'inventer une autre vie. C'est du moins ce que nous apprend la fin du roman telle qu'elle est dictée par l'expertise judiciaire représentée. Vous n'avez pas tué votre psychanalyste, mais il était plus attrayant de vous convaincre du contraire. Le récit n'est en somme que le résultat d'une bifurcation de l'esprit du personnage, manière de mettre du désordre dans l'ordre pour choquer le réel.

Selon René Girard (1978), pour que notre société fonctionne, l'organisation communautaire doit se baser sur un "mimétisme d'appropriation » qu'il décrit comme une contagion dans les rapports humains. Il faut donc se ressembler pour vivre ensemble et, dans ce mimétisme, on cherche à se retrouver dans l'image de l'autre. On exclut tous ceux qui sont inadaptés à la structure mimétique, ceux qui refusent de se prêter au jeu de l'imitation : on les déshumanise pour justifier qu'on les sacrifie (on les place dans un asile, on les brûle sur le bûcher), et ce sacrifice devient cathartique : les écarter de la scène sociale permet de s'immuniser contre la folie qu'ils représentent. Car la folie est contagieuse, selon la théorie de Girard. Les «femmes folles », pour revenir au roman de Julia Deck et à cette image qu'il critique, représentent toujours un danger pour l'ordre social, voire pour l'ordre 
narratif, car les « folles » incarnent la possibilité de ne pas se conformer, de ne pas imiter, un désir qui se trouve en réalité en chacun de nous : d'où l'importance de les sacrifier. D'où le sacrifice auquel consent la narration du roman en orchestrant la folie du personnage. Le «fou » est un bouc-émissaire qui sauve la communauté pour Girard. Dans Viviane Élisabeth Fauville, la « folle » est un bouc-émissaire. On la constitue meurtrière, et lorsque que la justice en décide autrement, elle regagne l'ordre. Son innocence proclamée, elle peut réintégrer les rangs.

Dans Femmes psychiatrisées, femmes rebelles et dans Women and Madness, Martine Delvaux et Phyllis Chesler montrent comment l'association entre " folie " et « femmes » s'effectue très rapidement dans nos sociétés, puisque la pression de se conformer, d'imiter, est beaucoup plus intense chez les femmes que chez les hommes. Dans les schèmes de la binarité sexuelle, la raison est associée au masculin, la folie au féminin. L'homme représente l'ordre, la femme le chaos. Le roman de Julia Deck n'est pas sans reprendre ces schèmes en les radicalisant. Ainsi, les représentants de la loi, de l'ordre, dans l'œuvre, sont tous des hommes et ils déversent leurs formules paternalistes. Et cela commence par le nom; bien qu'elle vienne d'être délaissée par son mari, on réduit Viviane à son nom comme on la réduirait au néant: " comment ça va, ma petite madame Hermant? 》(DECK, 2012 : 58); «Ce n'est pas la bonne tactique, madame Hermant, de jouer les imbéciles avec moi. » (DECK, 2012 : 59); "Certes, je pourrais imaginer, vu que vous passiez votre vie chez ce médecin, que vous êtes complètement cinglée. » (DECK, 2012 : 59.) Viviane est ce personnage exemplaire qu'une narration accuse, qu'une justice met sous silence, qu'un hôpital garde derrière les barreaux. La " chosification des patientes » en milieu psychiatrique, comme la conçoit Martine Delvaux, passe par une «objectivation du corps et de l'être de la "folle" » (DELVAUX, 1998 : 63). Dans le même esprit, Viviane est traitée, par le relais des narrations au pluriel ou impersonnelles, comme une femme parmi les femmes, ou plus manifestement, comme un personnage-marionnette dont la volonté ne dépend pas de ses moyens. En somme, elle n'a pas de moyen concret d'échapper à la situation qui l'accapare, elle est sans choix, malgré ce que le psychanalyste tente de lui faire croire : "Pourquoi avez-vous fait ce choix? / Je n'ai fait aucun choix, c'est mon mari qui m'a quittée. / Mais nous faisons tous des choix inconscients. / Vous suggérez que je l'ai poussé vers la porte. / Je ne suggère rien, c'est vous qui le dites. » (DECK, $2012:$ 22-23.) Une telle répartie, pernicieuse, accule à l'impasse (au silence) : la femme n'a pas choisi son sort, l'homme lui assure que oui, après quoi il ne lui laisse pas le choix : «Soyez gentille, recommencez le traitement » (DECK, 2012 : 23). Or, le roman Viviane Élisabeth Fauville pose une question que Martine Delvaux aborde aussi dans son ouvrage, à savoir : si on cherche à faire taire les «femmes folles », qu'arrive-t-il lorsque ces corps chosifiés se mettent à parler comme c'est le cas de Viviane, qui tout à coup se réinvente, se révolte? « Gentille. Vous ne le serez plus jamais. » (DECK, 2012, p. 23.) 
En réalité, la narration, comme nous l'avons mentionné, restitue à Viviane sa gentillesse par la résolution judiciaire de l'enquête qui l'innocente. Par ce fait même, elle la condamne à reprendre son rôle de mère sans histoire, à redevenir soumise à la nécessité. Viviane ne peut alors s'en sortir qu'à l'aide du lecteur, grâce à une lecture qui dépasserait le stéréotype et choisirait de déjouer la fermeture du texte. Une lecture volontairement insoumise qui refuserait de réduire le personnage de Viviane à un diagnostic, refuserait de lui rendre sa gentillesse, de lui supprimer ses affects, tous les affects (être jalouse, blessée, lasse, en colère) qui auraient pu la pousser au meurtre, qui sauraient expliquer le meurtre mieux que la folie, mais auxquels la narration, multipliant les ellipses, ne lui donne pas droit. Il est possible, ainsi, par contagion, par transfert du désir d'insoumission au lecteur, que le personnage de Viviane en ressorte triomphante, gagne en indépendance, assume ses propres choix. La narration, par l'indétermination qu'elle instaure dans le texte, encourage, précisons-le, cette lecture insoumise. Parsemée de contradictions, mensongère, elle sème le doute, l'organise. Si elle exauce, pour qui se laisse convaincre par la fin du récit, le discours dominant, du moins elle expose les manques de sa logique par l'usage de procédés narratifs qui échappent à un système cohérent. De fait, si la narration, non fiable en définitive, nous signale ses inconsistances, pourquoi la fin du récit - qui innocente Viviane - serait plus crédible que le meurtre qu'on y présente au début? Le meurtre que Viviane perpètre pendant que l'enfant est laissée seule à la maison.

C'est curieux parce que, si Viviane est clairement qualifiée de «folle » dans le roman, elle remplissait pourtant parfaitement son rôle de femme bourgeoise, jusqu'à ce qu'elle se retrouve déçue. En ce sens, sa situation rappelle bien celle des femmes psychiatrisées décrites par Phyllis Chesler : « Like many women, they buried their own destinies in romantically extravagant mariages, in motherhood, and in approved female pleasure. [Their] symptoms [are] as possibly the only way they could (unconsciously) resist or protest their traditional "feminine" work - or overwork7. » (CHESLER, 2005: 63 et 68.) Ainsi, dès que Viviane adopte un comportement considéré comme inapproprié pour son genre, elle devient soupçonnable, potentiellement criminelle; c'est notamment lorsqu'elle devient violente envers Julien, son mari, qu'il appelle les policiers pour retirer les bons propos qu'il avait tenus sur elle à leur intention. Elle dérange parce qu'elle n'incarne plus la jeunesse et la beauté; on cherche, au travail, à se débarrasser d'elle en la mutant.En revanche, son statut de bourgeoise bien éduquée, raisonnable, l'immunise contre le soupçon. Il est difficile pour le corps judiciaire et policier de croire qu'elle est une meurtrière. La violence des femmes paraît inconcevable à cet égard. D’ailleurs, la première incohérence que détecte l'inspecteur dans le discours de Viviane concerne son rôle de mère. Pour l'homme, la femme se doit d'être irrépro-

\footnotetext{
${ }^{7}$ « Comme plusieurs femmes, elles enterrent leur propre destinée dans des mariages extravagants, dans l'expérience de la maternité, et dans les plaisirs typiquement féminins. Leurs symptômes sont probablement la seule façon dont elles pourraient (inconsciemment) résister ou protester contre leur travail "féminin" traditionnel - ou le surmenage. » (Notre traduction.)
} 
chable : " comment aurait-elle pu laisser son enfant seule dans l'appartement " (DECK, 2012 : 163). À la fin du roman, on répare en conséquence la trame de l'action : "Vous étiez avec votre fille, que vous n'alliez tout de même pas laisser sans surveillance à douze semaines. » (DECK, 2012 : 152.)Une police règne dans le récit qui intercepte ce qui remue. Ainsi l'explique Martine Delvaux : "Appelée à se conformer aux normes, aux rôles, aux attentes qui déterminaient son sexe, aussitôt qu'un de ces rôles se trouvait transgressé, la femme devenait l'objet de la police "sexuelle" dont le rôle consistait à la remettre dans le droit chemin en l'exilant de la société. » (DELVAUX, 1998 : 32.) Ici, cela consiste à lui redonner son enfant et à la retourner à la maison, là où l'attendent ses besognes quotidiennes.

Or, que nous dit que la réalité de la fiction n'est pas autre? Après avoir essuyé des commentaires injurieux de la part de son psychanalyste, Viviane décide de commettre un acte qui n'est justement pas associé à son sexe, et ce, d'une part, afin de sortir du moule dans lequel on essaie de la tasser; d'autre part, afin de faire taire son psychanalyste, de le renvoyer au silence. De fait, même quand "vous » avez le couteau dans la main et que vous le menacez, le docteur ne vous croit pas capable de devenir meurtrière :

Le docteur sourit toujours, attendant la suite comme s'il était au spectacle. Bien sûr, de cela non plus il ne vous croit pas capable. Il n'a jamais vu en vous qu'une bourgeoise, une pâle carriériste, une névrosée de base qu'on domestique à coups de pilules blanches ou bleues. Il va enfin prendre la mesure de ce que vous êtes. (DECK, 2012 : 23.)

À plusieurs endroits dans l'œuvre sont semées des remarques qui rendent compte de l'oppression que subit Viviane en tant que femme, indices face auxquels le lecteur fait la sourde oreille s'il s'en tient au diagnostic de la folie. Il faut également noter le sexisme ordinaire dont le personnage féminin est victime. Un passage résume avec justesse la réflexion féministe qui est menée dans le texte : "Vous n'en tirez aucune fierté, et vous n'irez pas rapporter gaiement cette scène à votre fille âgée de huit ou neuf ans lorsqu'elle s'avisera de vous prendre en défaut, ayant établi par comparaison avec les volumes de la Bibliothèque rose que vous n'êtes pas la mère idéale vantée par les romans de bonne moralité. » (DECK, 2012 : 19.)C'est d'ailleurs la figure de la mère qui, dans le roman de Deck, fonctionne comme une brèche pour la lecture réparatrice que nous effectuons. Cette figure, constatons-nous, empêche le récit de se clore sur lui-même; elle ouvre la finale du roman, qui paraît autrement fermée, à un dernier doute possible.

Selon Phyllis Chesler, la folie féminine trouve toujours ses origines dans un rapport complexe à la mère. Dans Viviane Élisabeth Fauville, le rapport de Viviane à sa mère est insolite : elle conserve religieusement l'appartement de sa mère sans que personne n'y habite. Sa mère devient d'ailleurs son alibi; on peut lui de- 
mander de témoigner puisqu'elle a appelé Viviane, qui était à la maison avec sa fille le soir du meurtre. Pourtant, les recherches de la police leur permettent de conclure que cette mère est morte il y a deux ans. Les deux ans correspondent à la durée du mariage avec Julien. Le cadeau de mariage : un ensemble de couteaux qui serviront à tuer le psychanalyste. Enfin, la mère est toujours présente à la fin du récit; c'est elle qui ramène Viviane à la maison au sortir de l'hôpital, une fois que celle-ci est « guérie ». Aussi, la mère est une figure à la fois omniprésente et fuyante. On ne peut statuer définitivement sur son existence : est-elle morte ou vivante? Le fait qu'elle réapparaisse dans le dénouement, alors que les autorités policières ont donné la preuve de son décès, rouvre la question de la fiabilité de la narration. À quel moment la narration cesse-t-elle d'être retorse? S'agit-il de se rallier à la folie du personnage? S'agit-il de donner au lecteur les moyens de porter ses propres conclusions? Le texte, en effet, écrit Paul Ricœur, " comporte des trous, des lacunes, des zones d'indétermination, voire [...] met au défi la capacité du lecteur de configurer lui-même l'œuvre que l'auteur semble prendre un malin plaisir à défigurer. » (RICEUR, 1983: 145-146.) Et le défi, il est là, lancéau lecteur, à même l'impératif et l'adresse indirecte: "Gentille. Vous ne le serez plus jamais. » (DECK, 2012 : 23.) Ensuite, il suffit de faire un choix.

\section{LECTURE COMPENSATOIRE}

Chez Sophie Bienvenu, dans Et au pire, on se mariera, la narration est attribuée à l'enfant; l'enfant incapable de percevoir avec discernement le monde et les incidences des actes que l'on pose. L'enfant qui s'accroche à la fiction pour se donner l'illusion d'une vie pleine, susceptible de lui offrir contentement, et finalement qui participe à la formation de son image, fracturée, un peu floue, fragile. C'est cette image que capte l'auditeur, avons-nous voulu montrer, tandis qu'il gère, organise, sélectionne les informations qu'il reçoit et celles qui lui manquent forcément. Il manque la voix de la justice, nous l'avons dit. Elle est omniprésente et oriente le discours, mais n'est admise que par son silence. Ainsi est-elle souveraine, car elle arrive à influencer la parole de l'autre et le jugement du lecteur par sa seule autorité, mais en même temps inapte à se construire, elle, une sensibilité. Elle est alors réduite à une instance figée, froide, obscure. De ce manque surgit une parole, celle de l'enfant qui s'invente, d'autant plus humaine qu'elle porte en son sein les tensions qui habitent aussi l'auditeur, la faillibilité, le désir du secret mais la volonté de se raconter; la tension entre dissimulation et dévoilement qui appelle, selon nous, l'empathie de l'auditeur.

Avec La puissance des mouches, de Lydie Salvayre, nous nous trouvons en présence d'un personnage tout autrement constitué. L'homme qui narre se connaît par cœur, ne semble pas en train defabuler; plutôt il fabrique un discours, évite les chausse-trappes, se détourne de son devoir, n'assistera pas à son procès. La justice 
représentée dans l'œuvre, encore une fois, occupe un rôle central : malgré son absence, elle reçoit la parole. Or, cette parole, croyons-nous, fait le choix de ne pas s'adresser à elle, d'en dépasser l'autorité au moins par le biais de la narration, car il est impossible de la dépasser autrement. C'est la justice qui condamne, c'est elle qui emprisonne. Ainsi, le narrateur de La puissance des mouches, par ses digressions émotives, rationnelles, séduisantes, invite plutôt l'auditeur à s'identifier à lui, à comprendre ses motivations, à partager ses affects. Le lecteur, qui ne peut pas agir directement dans la fiction en se servant du marteau des juges, permet alors au personnage de ne pas seulement être un criminel. Avec ce roman, en ce sens, nous nous sommes permises une courte hypothèse survoltée; le lecteur, avons-nous risqué, pourrait admettre dans sa lecture empathique - à la suite du narrateur et s'appuyant sur ses confessions troublantes - le meurtre du père comme légitime vengeance. Les révélations qui concernent la violence du père ainsi que le silence des instances judiciaires nous incitent, en effet, à une lecture qui contrecarre la justice ou, en d'autres mots, choisit d'en ignorer le verdict. Le narrateur lui-même, de fait, ne cesse de se détourner de l'affaire qui occupe le récit, à savoir le procès, et partant, il ne cesse d'éviter le sujet de sa culpabilité. Ce détournement en appelle un autre que nous aimons à encourager pour tenter de soulever les voiles du texte, pour en dégager les zones d'ombre, soit le détournement interprétatif ou, dans les mots d'Umberto Eco (1996), la surinterprétation.

Ainsi qu'Eco l'explique, « nous procédons de la sorte parce que chacun de nous a introjecté en lui que d'un certain point de vue toute chose possède des relations d'analogie, de contiguïté et de similitude avec n'importe quelle autre » (ECO, 1996 : 44, l'auteur souligne).La parenté entre les choses qui paraissent significatives dans la lecture explique qu'une interprétation verse dans la paranoïa. Nous pensons que c'est ce qu'engendre particulièrement le roman Viviane Élisabeth Fauville de Julia Deck, dans lequel la « folie », justement, devient un mécanisme surpuissant. Si le stéréotype de la «femme folle » suit le personnage de Viviane à la trace, la narration plurielle se rallie au diagnostic au point de bouleverser tous les repères de lecture en multipliant les indices, réduisant par le fait même leur efficacité. Le lecteur, néanmoins, devant cet éclatement, surestime l'importance des indices, leur attribue un sens nouveau, les plie à son soupçon. Car il y a soupçon, dans Viviane Élisabeth Fauville : tout dans l'indétermination de la voix narrative, des événements de la fiction, de l'état psychique du personnage, amène le lecteur à élaborer une configuration sémantique qui lui permette, non pas, cette fois, d'humaniser le personnage, comme ce fut le cas pour les deux œuvres précédentes, mais de réparer le préjudice. La narration nous présente une femme criminelle pour l'innocenter; les spécialistes l'innocentent pour la rendre « folle». Cette « folie » exposée, la narration s'en amuse, de toute évidence. Elle travaille le stéréotype en donnant à la justice, aux hommes de loi, le juste mot sur les choses : les policiers notamment n'ont de cesse de rabaisser Viviane, de remettre en doute son récit - incohérent selon eux -, si bien qu'elle est réduite au silence, elle est 
réduite à leur jugement. Le roman de Deck fonctionne alors, croyons-nous, comme si la narration choisissait de faire contrepoids à la justice des hommes en brouillant les cartes pour offrir au lecteur un lieu propice au doute. Saisissant ce doute, nous avons voulu effectuer cette lecture réparatrice qui efface le préjudice et accepte qu'une femme ne soit pas « folle », et qui plus est, qu'elle assassine.

Telle qu'elle est représentée dans les romans de Sophie Bienvenu, de Lydie Salvayre et de Julia Deck, la justice fait défaut. Ou bien elle est manquante, effacée du texte et alors son silence dérange. Ou bien elle est défaillante, c'est-à-dire qu'elle échoue dans l'exercice de ses fonctions; elle échoue à reconnaître et respecter les droits de chacun, elle relance les stéréotypes, exerce une domination contestable. Ce défaut de justice, exposé par des narrations qui fabulent, se dispersent ou s'affolent, devient, d'une certaine manière, le nœud de la lecture. Car le lecteur de ces trois romans au cœur desquels se perpètre un crime est prié, en quelque sorte, de prendre parti; il cherche, du moins, une certaine vérité. Aussi est-il appelé à soupeser la tension qui règne entre justice et narration. Deux autorités s'affrontent, de fait, dans les œuvres, qui détiennent chacune leur vérité sur la fiction : l'autorité judiciaire, qui influence l'auditeur en raison de l'expertise qui lui est associée, puis l'autorité narrative, qui est celle-là fragile, toujours susceptible d'être remise en cause. Néanmoins, il nous semble que- et c'est ce que l'article a voulu montrer -, malgré les narrations troubles, déformantes ou démultipliées, c'est bien plutôt la justice, ici, qui est remise en cause. Du point de vue qui est le nôtre, le défaut de justice est à la fois attaqué et compensé par des narrations qui exposent leurs propres lacunes, signalant ainsi au lecteur qu'il vaudrait mieux ne pas lire « les yeux fermés ». Les romans à l'étude présentent, à cet effet, des narrations compensatoires, qu'on se «bricole», tel que l'exprime Laurent Demanze (2017), pour transcender la méfiance. Compensatoires dans la mesure où cohabitent en elles le sensible et l'indétermination, signaux d'alerte qui ne peuvent laisser le lecteur impassible. La fonction compensatoire lui est d'ailleurs léguée par les narrations; c'est ce que l'étude a permis de mettre au jour. Le lecteur compense à partir du moment où il est interpellé par des narrations qui s'adressent à lui de biais, par l'entremise d'un allocutaire muet ou par l'invitation indirecte, et dès lors il se retrouve en possession d'une force plus vive encore que la justice. Il lui est possible, s'il choisit la désobéissance, la lecture insoumise, de rendre au personnage, à l'accusé, à la victime - placés dans des cases, étiquetés par la justice - leur plein droit d'expression, d'humanité, d'existence. Même si, ne nous méprenons pas, il s'agit encore de fiction. 
BIENVENU, S.Et au pire, on se mariera. Montréal : La mèche, 2015 (2011).

BUTOR, M. Répertoire 1, tome II. Euvres complètes sous la direction de Mireille Calle-Gruber. Paris : Éditions de la Différence, 2006 (1964).

CHESLER, P. Women and Madness. New York : Palgrave Macmillan, 2015 (1972).

DECK, J. Viviane ÉlisabethFauville.Paris : Minuit, 2012.

DELVAUX, M. Femmes psychiatrisées, femmes rebelles. De l'étude de cas à la narration autobiographique. Paris : Institut Synthélabo, 1998.

DEMANZE, L. "Petit éloge de la paranoïa. Usages déréglés de la contreenquête ». Dans temps zéro (incursions), 31 mars 2017.Disponible en ligne sur $<$ http://tempszero.contemporain.info/document1532>. Accès le 2 juin 2017.

DOLEŽEL, L. Heterocosmica. Fiction and Possible Worlds. Baltimore (Londres) : Johns Hopkins University Press (Parallax : Re-visions of Culture and Society), 1998.

DUCROT, O.«Énonciation », Encyclopædia Universalis. Disponible en ligne sur $<$ http://www.universalis.fr/encyclopedie/enonciation/>. Accès le 2 juin 2017.

ECO, U.« La surinterprétation des textes ». Interprétation ou surinterprétation. Traduction de l'anglais par Jean-Pierre Cometti. Paris : Presses Universitaires de France, 1996 (1992).

GIRARD, R. Des choses cachées depuis la fondation du monde. Paris : Grasset, 1978.

NÜNNING, A. «Reconceptualizing Unreliable Narration : Synthesizing Cognitive and Rhetorical Approaches ». James Phelan et Peter J. Rabinowitz [dir.], A Companion to Narrative Theory. Malden/Oxford : Blackwell Publishing, p. 89-107.

OLSON, G.« Reconsidering Unreliability: Fallible and Untrustworthy Narrators ». Dans Narrative, vol. 11, no 1 (janvier), p. 93-109.

RICOEUR, P. Temps et récit I. Paris : Seuil, 1983. 
RIGGAN, W. Picaros, Madmen, Naïfs, and Clowns: The Unreliable First-Person Narrator. Norman : University of Oklahoma Press, 1981.

SALVAYRE, L. La puissance des mouches. Paris : Seuil (Points), 1995.

SCHUEREWEGEN,F. « Le texte du narrataire ». Dans Texte, no 5/6 (1986), p. 211-223. 